\title{
A STRICT KINETIC SOLUTION OF THE FINITE LENGTH MICROCHANNEL FLOW PROBLEM
}

\author{
Ching Shen \\ High Temperature Gas Dynamics Laboratory \\ Institute of Mechanics, Chinese Academy of Sciences \\ 15 Zhongguancun Road, 100080, Beijing, China \\ Tel 861062623973, Fax 861062561284, E-mail cshen@imech.ac.cn
}

\begin{abstract}
In finite length tube or channel gas flow the pressure gradient is determined by the global mass conservation law. In the continuum and slip flow the pressure distribution determined by the global mass conservation is given analytically. In the transitional flow regime an equation containing the flow rate of the Poiseuille flow solved by the strict kinetic theory is obtained and is shown to be the degenerated Reynolds equation for the lubrication theory. The integration of the equation is illustrated in the case of full diffuse reflection of the channel wall. The pressure distribution thus obtained is shown to be in excellent agreement with experimental data of long microchannels and the simulation results of the information preservation method. The results as having the strict kinetic theoretical merit are used to confirm the unfeasibility of the Lattice Boltzmann method in the transitional flow regime.
\end{abstract}

Keywords : microchannel flow, pressure distribution, MEMS, information preservation (IP) method, Lattice Boltzmann method (LBM)

\section{INTRODUCTION}

Microchannel is the basic constituent of the MEMS devices, its geometric form is regular and simple, but the gas flow in it is the basic flow pattern in MEMS and can reveal the specific features of the low speed micro internal flows, so it has been a subject of growing intensive investigation. In the classical fluid mechanics the steady Poiseuille flow is considered as a flow in long tube or channel having constant pressure gradient $\mathrm{G}=-\partial p / \partial x$ (see, e.g., Batchelor [1]). In the kinetic theoretical consideration a constant pressure gradient is also proposed for the linearized Boltzmann solution of the Poiseuille flow of gas (see e.g. Ohwada et al. [2]). But the constancy of pressure gradient in a tube or channel is true only for the flow of liquid (with constant density) and, as shown in the following, in any realistic tube or channel gas flow the pressure gradient is never a constant but varies from one cross section to another and is determined by the global mass conservation law. The assumption of the constancy of the pressure gradient in the Poiseuille flow is not necessary, in fact, the solution of the Poiseuille flow determines only the local flow characteristics in dependence of the local pressure gradient. As it is shown in the following the global mass conservation defines the pressure distribution in the continuum flow regime and the slip flow regime. In the transitional flow regime a strict kinetic theoretical solution of the channel flow pressure distribution is also provided by the mass conservation across the channel, given the kinetic theoretical solution of the mass flux for the Poiseuille flow is known. The channel pressure distribution so determined is compared with the experimental data of Pong et al. and Shih et al.[3,4] and the numerical simulation result by the information preservation (IP) method [5]. It is used also to test the validity of the calculation of the channel flow by the Lattice Boltzmann method accomplished by Nie et al. [6].

\section{NOMENCLATURE}

$\mathrm{h}$ : height of the channel

$K n$ : Knudsen number

L: length of the channel

$p$ : pressure

$P:=p / p_{0}$, dimensioinless pressure

$Q_{m}$ : mass flow rate

$\tilde{Q}_{m}$ : dimensionless mass flow rate

$R$ : gas constant

$T$ : temperature

$u$ : $x$ direction velocity

$x$ : Cartesian coordinate in the channel axis direction

$y$ : Cartesian coordinate normal to the channel surface

$z$ : the third Cartesian coordinate

$\varsigma:$ slip coefficient, $=(2-\sigma) \lambda / \sigma$

$\varsigma_{o}: \varsigma_{o}=\varsigma p=(2-\sigma) \lambda_{o} p_{o} / \sigma$

$\lambda:$ mean free path

$\mu:$ viscosity

$\rho:$ density 


\section{$\sigma: \quad$ tangential reflection coefficient}

subscript

$C$ : continuum flow

i: inlet

o; outlet

$S L I P:$ slip flow

$T R$ : transitional flow

\section{The pressure distribution determined by the global mass conservation}

Consider the gas flow in a channel between two plane plates at a cross section $x$ with the local pressure gradient $d p / d x$, which can vary from one cross section to another (see Fig.1). First we consider the continuum flow case. In the case of low speed the temperature variation is neglected, $\mu$ is a constant, the Navier-Stokes equation attains the form

$$
\frac{d^{2} u}{d y^{2}}=\frac{d p / d x}{\mu} \text {. }
$$

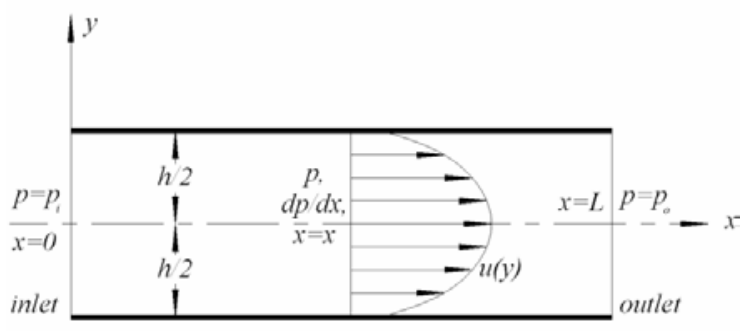

Fig. 1 The schematic of the Poiseuille flow

Equation (1) with the slipless boundary condition $u=0$ at $y=h / 2$ yields

$$
u=-\frac{1}{8 \mu}\left(h^{2}-4 y^{2}\right) \frac{d p}{d x} .
$$

The mass flux $Q_{m, C}$ flown in unit time through the gap between the two plates (with unit length in $z$ ) is obviously

$$
Q_{m, C}=2 \rho \int_{0}^{\frac{h}{2}} u d y=\frac{-p}{R T} \frac{d p}{d x} \frac{h^{3}}{12 \mu} .
$$

The global mass conservation across any cross section requires that

$$
p(d p / d x)=\text { constant },
$$

or in the dimensionless variables $P=p / p_{0}$ and $X=x / L\left(\left(p_{o}\right.\right.$ : the pressure at the outlet, $L:$ the length of the channel)

$$
P d P / d X=\text { constant. }
$$

From the above equation with the inlet and outlet boundary conditions

$P=P_{i} \equiv p_{i} / p_{o}$ at $X=0$, and $P=1$ at $X=1$,

the pressure distribution is easily determined

$$
P=P_{i}\left[1-\left(1-\frac{1}{P_{i}^{2}}\right) X\right]^{1 / 2},
$$

with the pressure gradient

$$
\frac{d P}{d X}=-\frac{1}{2} P_{i}\left(1-\frac{1}{P_{i}^{2}}\right)\left[1-\left(1-\frac{1}{P_{i}^{2}}\right) X\right]^{-1 / 2} .
$$

Obviously, the pressure distribution is approximately linear if the value $\left(1-1 / P_{i}^{2}\right)$ is small, and its slope is negative with $\mathrm{P}_{\mathrm{i}}^{2}>1$, and the absolute value of the pressure slope monotonously increases with increasing $X$.

In the case of slip flow the basic equation (1) remains the same, the velocity profile in the case of slip boundary condition

$$
\begin{gathered}
\left.u\right|_{y=h / 2}=-\zeta \frac{d u}{d y}, \text { with } \zeta=\frac{2-\sigma}{\sigma} \lambda, \quad \text { is } \\
u=-\frac{1}{8 \mu}\left(h^{2}-4 y^{2}+4 \zeta h\right) \frac{d p}{d x} .
\end{gathered}
$$

And the mass flux instead of Eq. (3) is obtained as

$$
Q_{m, S L I P}=2 \rho \int_{0}^{\frac{h}{2}} u d y=\frac{-p}{R T} \frac{d p}{d x} \frac{1}{4 \mu}\left(\frac{1}{3} h^{3}+2 \zeta h^{2}\right) \text {. }
$$

Note that $\varsigma$ is dependent on $p: \varsigma=(2-\sigma) \lambda / \sigma$, and as $\rho \lambda=$ constant, one can write $\varsigma=\varsigma_{o} / p$, where

$$
\varsigma_{o}=\frac{2-\sigma}{\sigma} \lambda p=\frac{2-\sigma}{\sigma} \lambda_{o} p_{o} .
$$

. So from Eq. (10) the mass conversation can be written as

$$
p \frac{d p}{d x}+6 \frac{\varsigma_{o}}{h} \frac{d p}{d x}=\text { constant }
$$

or in dimensionless variables

$$
P \frac{d P}{d X}+6 \frac{2-\sigma}{\sigma} K n_{0} \frac{d P}{d X}=\text { constant }
$$

where $K n_{o}=\lambda_{o} / h$ is Knudsen number at the outlet of the channel. The solution of the above equation with the integration constants determined from the inlet and outlet boundary conditions, Eq. (6), is

$$
\begin{aligned}
& P=-6 \frac{2-\sigma}{\sigma} K n_{o}+ \\
& \sqrt{\left(6 \frac{2-\sigma}{\sigma} K n_{o}\right)^{2}+P_{i}^{2}+12 \frac{2-\sigma}{\sigma} K n_{o} P_{i}+\left[1-P_{i}^{2}+12 \frac{2-\sigma}{\sigma} K n_{o}\left(1-P_{i}\right)\right] X}
\end{aligned}
$$

Note that the mass flux Eq. (10) in slip flow also can be written as

$$
Q_{m, S L I P}=\frac{-h^{3}}{12 \mu R T}\left(1+6 \frac{2-\sigma}{\sigma} K n\right) \frac{p d p}{d x},
$$

or

$$
Q_{m, S L I P}=\frac{-h^{3}}{12 \mu R T} \tilde{Q}_{m, S L I P} \frac{p d p}{d x} \quad, \quad \tilde{Q}_{m, S L I P}=1+6 \frac{2-\sigma}{\sigma} K n,
$$

if the first half of Eq. (11) is used for $\varsigma$, and here $K n=\lambda / \mathrm{h}$ is the local Knudsen number. This is the well known solution of the mass flow rate of the Poiseuille flow in the slip flow regime, where $p, d p / d x$ and $K n$ are local values of pressure, pressure gradient and Knudsen number.

The above expression of pressure distribution in the slip flow regime seems to be obtained the earliest by Arkilic 
through a different derivation as a first approximation with accuracy of order $\varepsilon=H / L$ (see Ph.D. Thesis of E. B. Arkilic [7] and its publication version [8]), and the similar results have been also published and recorded in $[9,10,11]$.

In the transitional flow regime the flow rate of the plane Poisuille flow has been solved by the linearized Boltzmann equation or its BGK [12] model version by many authors (see e.g. by Cercignani and Daneri [13] and Ohwada et al. [2]). Fukui and Kaneko [14] in derivation of the generalized Reynolds lubrication equation also calculated the flow rate of Poiseuille flow numerically, and later [15] they had used this flow rate calculated rigorously to generate a database for rapid calculation of the generalized Reynolds equation for high Knudsen numbers. The flow rate of the Poiseuille flow can be expressed in the form

$$
Q_{m, T R}=\frac{-h^{3}}{12 \mu R T} \tilde{Q}_{m, T R}(K n) \frac{p d p}{d x},
$$

where $K n$ is the local Knudsen number and $\tilde{Q}_{m, T R}(K n)$ is the flow rate in transitional regime (normalized by the slip-less value $Q_{m, C}$ ) calculated from the linearized Boltzmann equation for Poiseuille flow. A tabled database of the calculated values of $\tilde{Q}_{m, T R}(K n)$ for $\sigma=1, \sigma=0.9, \sigma=0.8$ and $\sigma=0.7$ is provided in [15], and a fitted formula approximation for diffuse reflection $(\sigma=1)$ by Robert is recorded in [16] (there the second term on the right hand side is misprinted as $6 A \sqrt{\pi} K n$ )

$$
\tilde{Q}_{m, T R}(K n)=1+6 A K n+\frac{12}{\pi} K n \log (1+B K n),
$$

where $A=1.318889$ and $B=0.387361$. The global mass conservation, i.e., the constancy of the mass flux being equal at any cross section, requires

$$
\tilde{Q}_{m, T R}(K n) \frac{p d p}{d x}=\text { cons } \tan t,
$$

or in the dimensional form

$$
\frac{d}{d X}\left[\tilde{Q}_{m, T R}(K n) P \frac{d P}{d X}\right]=0 .
$$

With the database incorporated this equation is valid for any surface conditions of the plates and can be integrated numerically. But for the illustrative purpose only the case of complete diffuse reflection, $\sigma=1$, is expounded here, then the mass conservation is (from Eqs. (17) and (19))

$$
\frac{d}{d X}\left\{\left[1+6 A K n+\frac{12}{\pi} K n \log (1+B K n)\right] P \frac{d P}{d X}\right\}=0 .
$$

For the ease of integration the local Knudsen number $K n$ is most conveniently expressed through $P$

$$
K n=\frac{\lambda}{h}=\frac{\lambda}{h} \frac{p_{o}}{p}=K n_{o} / P,
$$

and Eq. (20) becomes

$$
\left[P+6 A K n_{o}+\frac{12}{\pi} K n_{o} \log \left(1+\frac{B K n_{o}}{P}\right)\right] \frac{d P}{d X}=D,
$$

where $D$ is an unspecified constant to be determined from the integration and has the physical meaning of the flow rate across the channel normalized by the slip-less flow rate value. The equations determining the pressure distribution (19) and (20) are in fact the generalized Reynolds equation of the gas lubrication theory degenerated for the microchannel flows (see $[17,18])$. They are integrated with the inlet and outlet boundary conditions (6) to yield the pressure distribution in the transitional regime.

\section{Comparison with the experimental data and the} simulation results

To compare the above results with the experimental data of flow in the microchannel with pressure sensors imbedded in the channel accomplished by Pong et al. [3] and Shih et al.[4], we calculate the pressure distribution for nitrogen in the $1.2 \times 40 \times 3000 \mathrm{\mu m}^{3}$ channel [3] and for helium in the $1.2 \times 40 \times 4000 \mu^{3}$ channel [4]. Under the experimental condition $T_{0}=294 K$ the value of $K n_{o}$ for nitrogen is 0.052325 , and for helium is 0.15579 . The experimental data of nitrogen is compared with the results of the global mass conservation in the continuum regime, Eq. (7), and the slip flow regime, Eq. (13), in Fig. 2. The pressures $p_{i}$ at the inlet of the channel in psig are given in [3] and [4], and the corresponding values of $P_{i}=\left.P\right|_{X=0}$ are listed in Table 1. In Eq. (13) $K n_{o}$ is taken equal to 0.052325 , and $\sigma=1$. The comparison shows that the global mass conservation predicts the pressure distribution quite well and the slip flow result improves the agreement of the continuum prediction with the experimental data. The experimental data are also compared with the prediction of the transitional flow regime. Equation (22) is integrated under the following boundary condition

$\left.P\right|_{X=0}=p_{i} / p_{o}$ as given in Table 1 , and $\left.P\right|_{X=1}=p_{o} / p_{o}=1$,

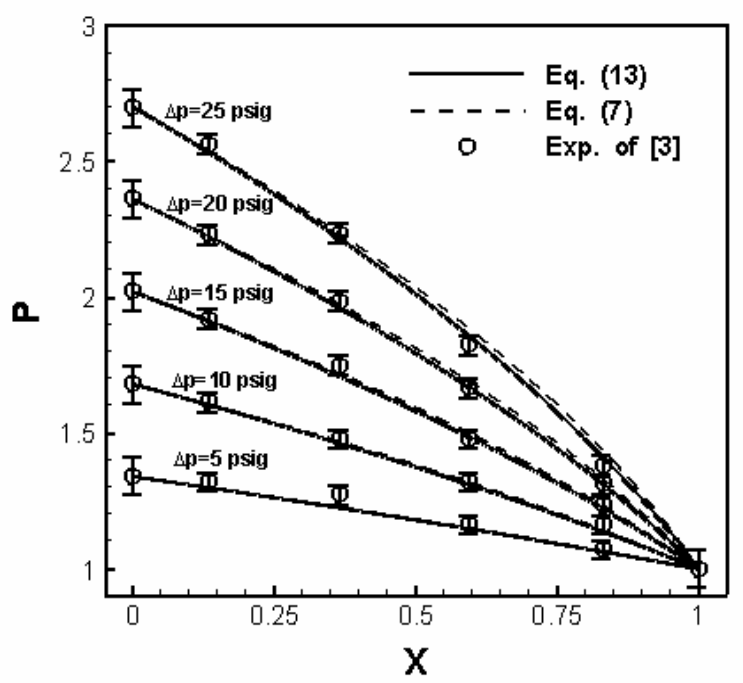

Fig. 2 Comparison of the pressure distribution obtained from slip solution, Eq.(13), (solid line) and slipless solution, Eq. (7), (dashed Line) with experimental data in a $1.2 \times 40 \times 3000 \mu^{3}$ microchannel for nitrogen [3] (symbols). $K n_{o}=0.052325$. 
Table I The experimental inlet pressure data in psig and corresponding values of $\left.P\right|_{X=0}$ for nitrogen and

\begin{tabular}{lrrrrr}
\multicolumn{5}{c}{ helium } \\
\hline for nitrogen $^{[3]}$ & & & & & \\
$p_{i} \quad$ (in psig) & 5 & 10 & 15 & 20 & 25 \\
$\left.P\right|_{X=0}$ & 1.3402 & 1.6805 & 2.0207 & 2.3609 & 2.7012 \\
for helium ${ }^{[4]}$ & & & & & \\
$p_{i} \quad($ in psig $)$ & 8.7 & 13.6 & 19.0 & & \\
$\left.P\right|_{X=0}$ & 1.5920 & 1.9254 & 2.2929 \\
\hline
\end{tabular}

The results of integration are presented in Fig.3 and Fig.4. It is seen from the figures that the results of the global mass conservation (degenerated Reynolds equation) agree well with the experimental data. The finite length channel flows under the experimental conditions have been calculated also by the information preservation (IP) method $[19,20]$ in Shen, Fan and Xie [21]. The IP simulation results are also presented in Figs.3 and 4 by dashed lines. The comparison shows excellent agreement between the results calculated by the global mass conservation and by the IP method.

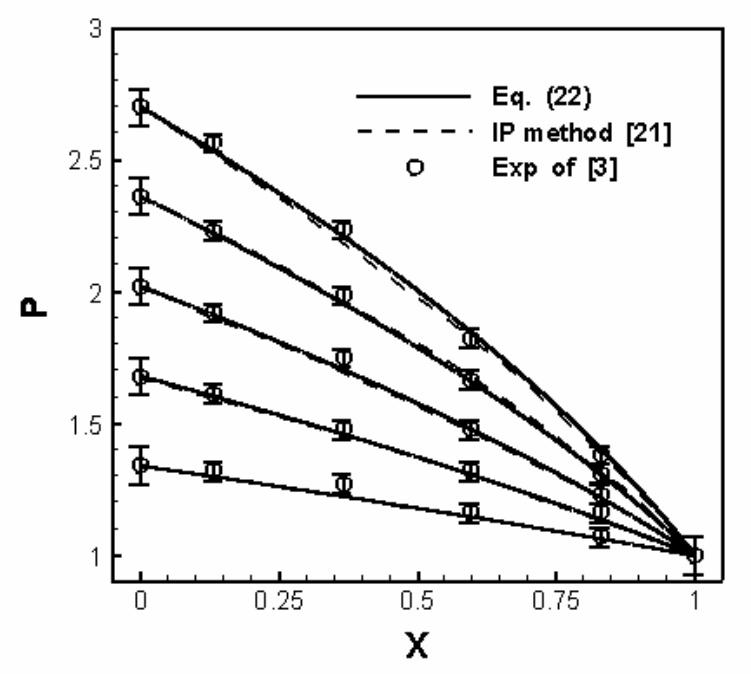

Fig.3 Comparison of the pressure distribution from Eq.(22) (solid line) with experimental data in a $1.2 \times 40 \times 3000 \mu^{3}$ microchannel for nitrogen [3] (symbols) and the result of IP method [21] (dashed line). $K n_{o}=0.052325$. See also [18].

\section{Examination of the feasibility of LBM in solving the microchannel problem in transitional regime}

The Lattice Boltzmann method (LBM, see [22] and references cited there) solves the simplified Boltzmann equation on lattice points. LBM solution converges to the Navier-Stokes solution for small $\mathrm{Kn}$. The ease of LBM in handling complex geometry, simplicity in implementation and its high efficiency makes it tempting to be used in simulating gas flows in MEMS. Recently Nie, Doolen, and Chen [6] attempt to use it in transitional regime.

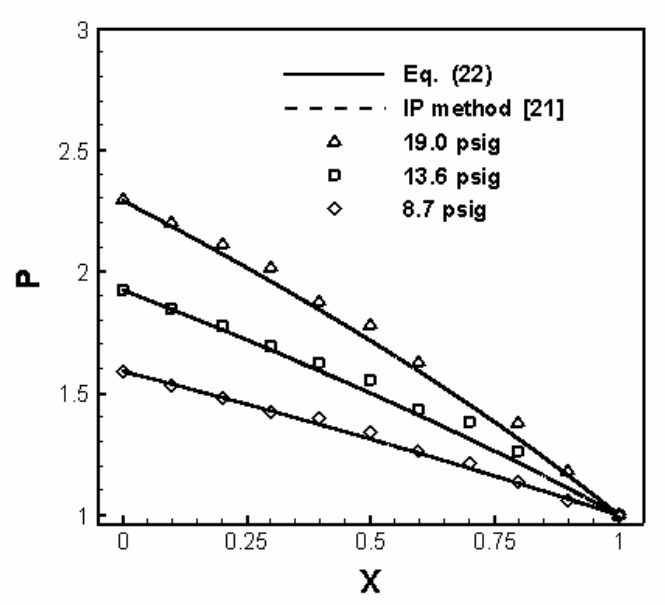

Fig.4 Comparison of the pressure distribution from Eq.(22) (solid line) in a $1.2 \times 40 \times 4000 \mu^{3}$ with experimental data in a microchannel for helium [4] (symbols) and the the IP method [21] (dashed lines, note that the solid lines and the dashed lines almost coincide). $K n_{o}$ $=0.15579$

It is of fervent concern for the scientific community to know whether LBM is capable to simulate correctly the transitional regime flows in MEMS. Shen, Tian, Xie and Fan examined the feasibility of using LBM in simulating MEMS flows by comparison with the DSMC calculations [23]. Here the LBM results are compared with the calculations by using the mass conservation equation or the degenerated Reynolds equation to attain the same conclusions as in Reference [23] but this time the conclusion is confirmed by a test stone with the merit of strict kinetic theory. The equation (22) is integrated under the following conditions (definitely in transitional regime) for a short $1 \times 100 \mu \mathrm{m}^{2}$ microchannel that have been considered by LBM in Nie, Doolen and Chen [6]

$K n_{o}=0.194,\left.P\right|_{X=0}=2,\left.P\right|_{X=1}=1$,

$K n_{o}=0.388,\left.P\right|_{X=0}=2,\left.P\right|_{X=1}=1$.

The results of comparison of results of equation (22) with those of Nie, Doolen and Chen by using the Lattice Boltzmann method (LBM) [6] are shown in Fig.5 and Fig.6. Also shown in the figures are the DSMC and IP results. From the comparison it is seen that the DSMC method, the IP method and the mass conservation equation (degenerated Reynolds equation) yield almost identical pressure distribution, but the LBM results are quite different from the calculations of the degenerated Reynolds equation and as well from DSMC and IP results, so it can be concluded that the version of LBM employed in Reference [6] is not feasible for simulating microchannel flows in the transitional regime. For the detailed examination of the feasibility of the LBM method in simulating transitional regime microchannel flows readers are referred to Reference [18] where among other things the version of LBM used [6] was briefly described. Also, there the comparison of the pressure distributions was given in the form of deviation from linearity, so the differences between results of various methods were revealed in more relief. In this paper the quantities being 
compared are the pressure distributions themselves and the same conclusions are achieved. But here the conclusion of unfeasibility of the LBM in simulating transitional flows is reconfirmed by the comparison with calculations having the strict kinetic theoretical merit.

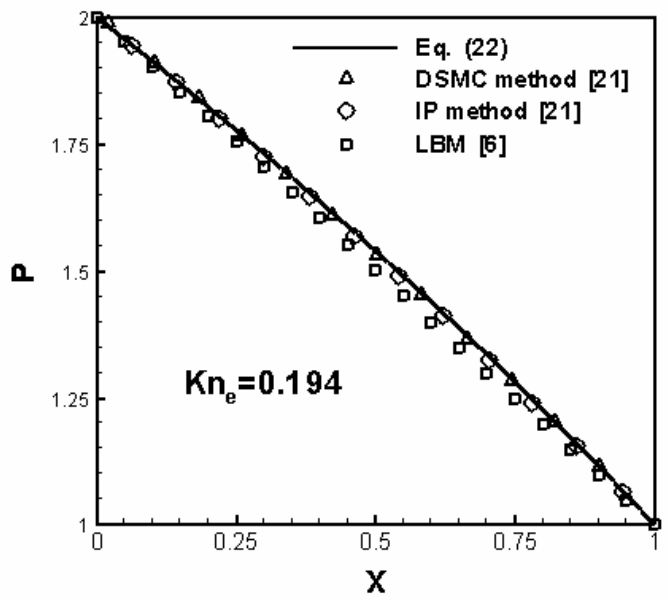

Fig. 5 Pressure distribution in a microchannel with $K n=0.194$ at outlet $(h / L=100)$. Comparison of equation (22), DSMC, IP methods [21] and the LBM method [6]

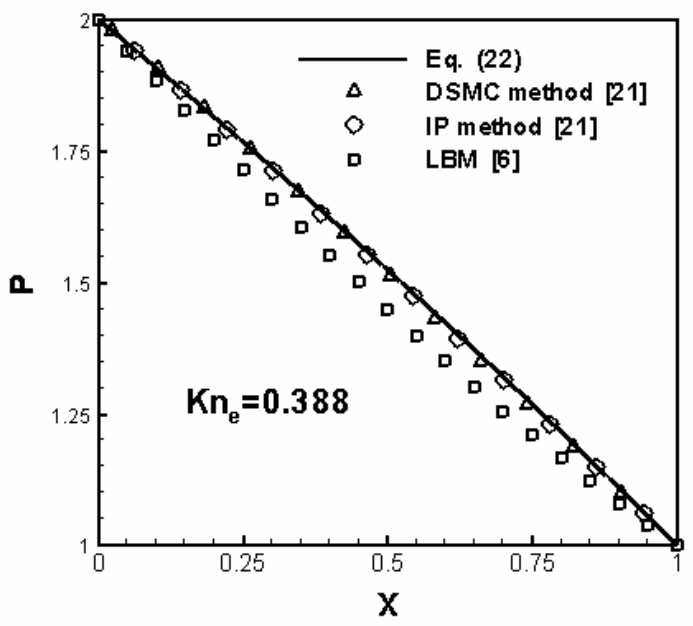

Fig. 6 Pressure distribution in a microchannel with $K n=0.388$ at outlet $(h / L=100)$. Comparison of equation (22), DSMC, IP methods [21] and the LBM method [6]

It is noted, that in [23] the comparison of LBM with DSMC and IP methods was also given for smaller Knudsen numbers (e.g., $K n=0.0194)$ where good agreement between them was obtained, so the failure of LBM in predicting microchannel flow is verified for transitional flow regime ( $K n=0.194$, 0.388 ) but not for slip flow regime. For the relative error of LBM readers are referred to [23], where the results were shown via the deviation $\left(p-p_{l}\right) / p_{o}$ of the pressure distribution from the linear distributed pressure $p_{l}=p_{0}+\left(p_{i}-p_{o}\right)(1-X)$.

\section{Concluding remarks}

In the present paper the finite length microchannel pressure distribution for continuum flow, slip flow and transitional flow regimes is determined by the global mass conservation equation. In the transitional flow regime this equation is the Reynolds equation for the gas film lubrication problem degenerated by the suggestion of the author for solving the microchannel flow [18]. The comparison shows excellent agreement with the experimental data and the IP results in the long microchannels. Earlier the IP results for long channel were only compared [5] with the experimental data (the DSMC was not able to accomplish simulation of the long channel flow where the experimental data were available), now they are verified by comparison with results having the strict kinetic theory merit. The global mass conservation equation (the degenerated Reynolds equation) provides a means with the merit of strict kinetic theory to test various methods in solving the micro scale rarefied gas dynamics flows in transitional regime. The unfeasibility of LBM for solving the transitional flow problem [23] is reconfirmed by this means. From the practical application point of view database for the flow rates of the Poiseuille flow with various combinations of possible surface properties obtained from strict kinetic theory is an actual task for the solution of the microchannel flow, and also for the thin film air bearing problem and the gas damping problem [24] in micromechanical accelerometers. The database in the form of fitting formulas is especially desirable.

\section{ACKNOWLEDGMENTS}

The support by the NNSFC (grant 90205024) is appreciated. The author also appreciates the help of Dr Jiang JZ in carrying the calculation and the drawings.

\section{REFERENCES}

1. Batchelor, G.K., 1967, An Introduction to Fluid Dynamics, Cambridge University Press, Cambridge.

2. Ohwada, T., Sone, Y. and Aoki, K, 1989, "Numerical analysis of the Poiseuille and thermal transpiration flows of a rarefied gas between two parallel plates," Phys. of Fluids A1, pp. 2042-2049.

3. Pong, K.C., Ho, C.M., Liu, J.Q., and Tai, Y.C., 1994, "Non-linear pressure distribution in uniform microchannels," ASME, FED 197, Application of Microfabrication to Fluid Mechanics, pp. 51-56.

4. Shih, J.C., Ho, C.M., Liu, J.Q., and Tai, Y.C., 1996, "Monatomic and polyatomic gas flow through uniform microchannels," ASME-DSC 59, pp. 197

5. Shen, C., Fan, J. and Xie, C., 2003, "Statistical simulation of rarefied gas flows in micro-channels," Journal of Computational Physics, 189, pp.512-526.

6. Nie, X., Doolen, G.D. and Chen, S., 2002, "Lattice Boltzmann simulation of fluid flows in MEMS," Journal of Statistical Physics, 107, pp.279-289.

7. Arkilic, E.B., 1997, "Measurement of the mass flow and tangential momentum accommodation coefficient in silicon micromachined channels", FDRL TR 97-1, Ph.D. Thesis MIT.

8. Arkilic, E.B. et al., 2001, "Mass flow and tangential momentum accommodation coefficient in silicon micromachined channels", J. Fluid Mech., 437, pp.29-43. 
9. Colin, S. "Rarefaction and compressibility effects on steady and transient gas flows in microchannels", Microfluidics and Nanofluidics, Review, published on line.

10. Elizalova, T.G., 2001, Comput. Mathem. and Mathem. Physics, 41, pp.219-234.

11. Graur, I.A., Meolans, J.G., Zeitoun, D.E., Gaseous slip flow in microchannels, $24^{\text {th }}$ RGD Symposium, Bari, Italy, July 10-16, 2004, Paper Th 10.

12. Bhatnagar, P.L., Gross, E.P. and Krook, M., 1954, "A model for collision processes in gases, I," Physics Review, 94, pp.511-525.

13. Cercignani C. and Daneri, A., 1963, "Flow of a rarefied gas between two parallel plates," Journal of Applied Physics, 34, pp.3509-3513.

14. Fukui, S. and Kaneko, R., 1988, "Analysis of ultra-thin gas film lubrication based on linearized Boltzmann equation: first report-derivation of a generalized lubrication equation including thermal creep flow," Journal of Tribology, 110, pp.253-262.

15. Fukui, S. and Kaneko, R.,1990, "A database for interpolation of Poiseuille flow rates for high Knudsen number lubrication problems," Journal of Tribology, 112, pp. $78-83$.

16. Alexander, F.J., Garcia, A.L. and Alder, B.J., 1994, "Direct simulation Monte Carlo for thin film bearings," Physics of Fluids, 6, pp. $3854-3860$.

17. Shen, C., 2005, Rarefied Gas Dynamics, Fundamentals, Simulations and Micro Flows, Springer Verlag, Berlin, Heidelberg, New York
18. Shen, C., 2005, Use of the degenerated Reynolds equation in solving the microchannel flow problem, accepted, to appear in Physics of Fluids, April issue of 2005.

19. Fan, J. and Shen, C., 1999, Statistical simulation of lowspeed unidirectional flows in transitional regime, in Rarefied Gas Dynamics, edited by R Brun, R Campargue, R Gatignol, J C Lengrand, Cepadues Editions, 2, pp. 245252.

20. Fan, J. and Shen, C., Statistical simulation of low-speed rarefied gas flows. Journal of Computational Physics, 167, pp.393-412.

21. Shen, C., Fan, J. and Xie, C., 2003, Statistical simulation of rarefied gas flows in micro-channels. Journal of Comp Physics, 189, pp.512-526.

22. Chen, S. and Doolen, G.D., 1998, Lattice Boltzmann Method for Fluid Flows, Annual Review of Fluid Mechanics, 30,329-364.

23. Shen, C., Tian, D. B., Xie, C. and Fan, J., 2003 , Examination of LBM in simulation of the micro-channel flow in transitional regime. Proceedings of the $1^{\text {st }}$ Intern. Conference on micro and mini-channels, April 2425,2003, Rochester, edited by S.G. Kandlikar, ASME, 405-410 (2003), see also Shen, C., Tian, D. B., Xie, C. and Fan, J., 2004, Microscale Thermophysical Engineering, 8, 423-432

24. T. Veijola, H. Kuisma, J. Lahdenpera, 1995, Equivalentcircuit model of the squeezed gas film in a silicon accelerometer, Sensors and Actuators, A48,239. 